\title{
GENERALIZED SCHUR COMPLEMENTS OF MATRICES AND COMPOUND MATRICES*
}

\author{
JIANZHOU LIU ${ }^{\dagger}$ AND RONG HUANG
}

\begin{abstract}
In this paper, we obtain some formulas for compound matrices of generalized Schur complements of matrices. Further, we give some Löwner partial orders for compound matrices of Schur complements of positive semidefinite Hermitian matrices, and obtain some estimates for eigenvalues of Schur complements of sums of positive semidefinite Hermitian matrices.
\end{abstract}

Key words. Löwner partial order, generalized Schur complement, compound matrix, eigenvalues.

AMS subject classifications. 15A45, 15A57.

1. Introduction. Many important results for compound matrices and Schur complements of matrices have been obtained in [2,3,9,10]. Recently, Smith [11], Liu et al. [5,6], Li et al. [4] obtained some estimates of eigenvalues and singular values for Schur complements of matrices. Liu and Wang [7], Wang and Zhang et al. [12] obtained some Löwner partial orders of Schur complements for positive semidefinite Hermitian matrices respectively. Wang and Zhang [13] obtained some Löwner partial orders for Hadamard products of Schur complements of positive definite Hermitian matrices. Liu [8] obtained some Löwner partial orders for Kronecker products of Schur complements of matrices. In this paper, we study Schur complements of matrices and compound matrices.

Let $\mathbb{C}, \mathbb{R}$ and $\mathbb{R}_{+}$denote the set of complex, real, and positive real numbers respectively. Let $\mathbb{C}^{m \times n}$ denote the set of $m \times n$ complex matrices. Let $N_{n}$ denote the set of $n \times n$ normal matrices. Let $H_{n}$ denote the set of $n \times n$ Hermitian matrices, and let $H_{n} \geq\left(H_{n}^{>}\right)$denote the subset consisting of positive semidefinite (positive definite)

*Received by the editors on June 10, 2009. Accepted for publication on July 31, 2010. Handling Editors: Roger A. Horn and Fuzhen Zhang.

${ }^{\dagger}$ Department of Mathematical Science and Information Technology, Hanshan Normal University, Chaozhou, Guangdong 521041, China; and Department of Mathematics and Computational Science, Xiangtan University, Xiangtan, Hunan, 411105, P. R. China. This work was supported in part by the National Natural Science Foundation of China 10971176, the Guangdong Provincial Natural Science Foundation of China 10152104101000008 and the key project of Hunan Provincial Natural Science Foundation of China 10JJ2002. E-mail: liujz@xtu.edu.cn

${ }^{\ddagger}$ Department of Mathematics and Computational Science, Xiangtan University, Xiangtan, Hunan. This work was supported by China Postdoctoral Science Foundation (No. 20090451103) and the Research Foundation of Education Bureau of Hunan Province (No. 09C942). 
Hermitian matrices. For $A \in \mathbb{C}^{m \times n}$, the rank of $A$ is denoted by $r(A)$. Denote by $A^{*}$ the conjugate transpose matrix of $A$. For $A, B \in H_{\bar{n}}^{\geq}$, write $B \geq A$ if $B-A \in H_{n}$. The relation " $\geq$ " is called the Löwner partial order. For $A \in \mathbb{C}^{n \times n}$, we always arrange the eigenvalues of $A$ as $\left|\lambda_{1}(A)\right| \geq \cdots \geq\left|\lambda_{n}(A)\right|$. For $A \in \mathbb{C}^{m \times n}$, denote the column space of $A$ by $\Re(A)$.

Let $k$ be an integer with $1 \leq k \leq n$. Define

$$
Q_{k, n}=\left\{\omega=\left\{\omega_{1}, \ldots, \omega_{k}\right\}: \omega_{i} \in \mathbb{R} \text { and } 1 \leq \omega_{1}<\cdots<\omega_{k} \leq n\right\} .
$$

Given a matrix $A=\left(a_{i j}\right) \in \mathbb{C}^{m \times n}$. Let $k$ and $r$ be integers satisfying $1 \leq k \leq m$ and $1 \leq r \leq n$, respectively. If $\alpha \in Q_{k, m}$ and $\beta \in Q_{r, n}$, then $A(\alpha, \beta)$ denotes the $k \times r$ matrix whose $(i, j)$ entry is $a_{\alpha_{i}, \beta_{j}}$. If $\alpha$ is equal to $\beta, A(\alpha \mid \alpha)$ is abbreviated to $A(\alpha)$. Let $A \in \mathbb{C}^{m \times n}, l=\min \{m, n\}, k \in L=\{1,2, \ldots, l\}$. We denote by $C_{k}(A)$ the kth compound matrix. Let all the elements of $Q_{k, m}$ be ordered lexicographically; " $"$ denotes the lexicographical order. Let $Q_{r, m}=\left\{\alpha_{i} \mid i=1, \ldots,\left(\begin{array}{c}m \\ r\end{array}\right)\right\}$ satisfy

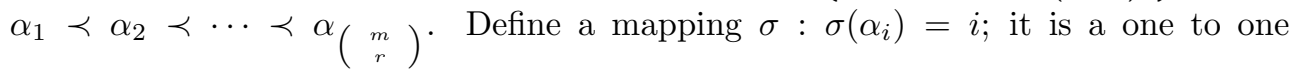
correspondence. We denote $\sigma(\alpha)$ by $j_{\alpha}$ if $\alpha \in Q_{r, m}$. If $\alpha \in Q_{r, m}$ and $\beta \in Q_{k, n}$, then $A_{j_{\alpha}, j_{\beta}}$ denotes the $\left(j_{\alpha}, j_{\beta}\right)$ entry of $C_{k}(A)$.

Let $A \in \mathbb{C}^{m \times n}$. If $X \in \mathbb{C}^{n \times m}$ satisfies the equations

$$
\text { (i) } A X A=A, \quad \text { (ii) } X A X=X, \quad(i i i)(X A)^{*}=X A, \quad(i v)(A X)^{*}=A X \text {, }
$$

then $X$ is called the Moore-Penrose (MP) inverse of $A$.

Let $A \in \mathbb{C}^{m \times n}, \alpha \subset M, \beta \subset N, \alpha^{\prime}=M-\alpha$, and $\beta^{\prime}=N-\beta$. Then

$$
A /+(\alpha, \beta)=A\left(\alpha^{\prime}, \beta^{\prime}\right)-A\left(\alpha^{\prime}, \beta\right)[A(\alpha, \beta)]^{+} A\left(\alpha, \beta^{\prime}\right)
$$

is called the generalized Schur complement with respect to $A(\alpha, \beta)$. If $A(\alpha, \beta)$ is a nonsingular matrix, then $A /{ }_{+}(\alpha, \beta)=A /(\alpha, \beta)$ is called the Schur complement with respect to $A(\alpha, \beta)$. If $\alpha=\beta$, we define $A /+(\alpha, \beta)=A /+\alpha$ and $A /(\alpha, \beta)=A / \alpha$ respectively. In [1], Ando shows that if $A, B \in H_{\bar{n}}^{\geq}$, then

$$
(A+B) / \alpha \geq A / \alpha+B / \alpha,
$$

and

$$
A^{\frac{1}{2}} / \alpha \geq(A / \alpha)^{\frac{1}{2}} .
$$

In this paper, we provide some similar results for compound matrices of the Schur complements of positive semidefinite Hermitian matrices and obtain some estimates for eigenvalues. 
2. Some formulae for compound matrices of generalized Schur complements of matrices. In this section, using properties of compound matrices and $M P$ inverses, we obtain some formulae for compound matrices of generalized Schur complements of matrices.

Lemma 2.1. Let $A \in \mathbb{C}^{m \times n}$. Then

$$
C_{k}\left(A^{+}\right)=\left[\left(C_{k}(A)\right]^{+} .\right.
$$

Proof. By properties of compound matrices, we have
i. $C_{k}(A)=C_{k}\left(A A^{+} A\right)=C_{k}(A) C_{k}\left(A^{+}\right) C_{k}(A)$,
ii. $C_{k}\left(A^{+}\right)=C_{k}\left(A^{+} A A^{+}\right)=C_{k}\left(A^{+}\right) C_{k}(A) C_{k}\left(A^{+}\right)$,
iii. $C_{k}\left(A^{+} A\right)=C_{k}\left(A^{+}\right) C_{k}(A)$,
iv. $C_{k}\left(A A^{+}\right)=C_{k}(A) C_{k}\left(A^{+}\right)$.

Thus, by equations (i)-(iv) of (1.2), we easily get that $C_{k}\left(A^{+}\right)=\left[C_{k}(A)\right]^{+}$. $\mathrm{u}$

Lemma 2.2. ([4]) Let $A \in \mathbb{C}^{m \times n}$ be partitioned as

$$
A=\left(\begin{array}{ll}
A_{11} & A_{12} \\
A_{21} & A_{22}
\end{array}\right)
$$

where

$$
r\left(\begin{array}{l}
A_{11} \\
A_{21}
\end{array}\right)=r\left(A_{11}, A_{12}\right)=r\left(A_{11}\right),
$$

and

$$
r\left(\begin{array}{l}
A_{12} \\
A_{22}
\end{array}\right)=r\left(A_{21}, A_{22}\right)=r\left(A_{22}-A_{21} A_{11}^{+} A_{12}\right) .
$$

Then

$$
A^{+}=\left(\begin{array}{cc}
A_{11}^{+}+A_{11}^{+} A_{12} S^{+} A_{21} A_{11}^{+} & -A_{11}^{+} A_{12} S^{+} \\
-S^{+} A_{21} A_{11}^{+} & S^{+}
\end{array}\right),
$$

where $S=A_{22}-A_{21} A_{11}^{+} A_{12}$.

Lemma 2.3. Let $A \in H_{n}^{\geq}$be partitioned as (2.2) with

$$
r(A)=r\left(A_{11}\right)+r\left(A_{22}\right) .
$$

Then (2.3) holds. 
Theorem 2.4. Let $A \in \mathbb{C}^{m \times n}, \alpha \subset M, \beta \subset N, \alpha^{\prime}=M-\alpha$ and $\beta^{\prime}=N-\beta$. Set $1 \leq k \leq \min \{|M-\alpha|,|N-\beta|\}$. Suppose that the following conditions are satisfied:

$$
\begin{aligned}
& r\left(\begin{array}{c}
A\left(\alpha^{\prime}, \beta^{\prime}\right) \\
A\left(\alpha, \beta^{\prime}\right)
\end{array}\right)=r\left(A\left(\alpha^{\prime}, \beta^{\prime}\right), A\left(\alpha^{\prime}, \beta\right)\right)=r\left(A\left(\alpha^{\prime}, \beta^{\prime}\right)\right), \\
& r\left(\begin{array}{c}
A\left(\alpha^{\prime}, \beta\right) \\
A(\alpha, \beta)
\end{array}\right)=r\left(A\left(\alpha, \beta^{\prime}\right), A(\alpha, \beta)\right)=r\left(A /_{+}(\alpha, \beta)\right) ;
\end{aligned}
$$

and

$$
r\left(\begin{array}{c}
C_{k}\left[A\left(\alpha^{\prime}, \beta^{\prime}\right)\right] \\
C_{k}(A)\left(\gamma, \delta^{\prime}\right)
\end{array}\right)=r\left(C_{k}\left[A\left(\alpha^{\prime}, \beta^{\prime}\right)\right], C_{k}(A)\left(\gamma^{\prime}, \delta\right)\right)=r\left\{C_{k}\left[A\left(\alpha^{\prime}, \beta^{\prime}\right)\right]\right\}
$$

$$
r\left(\begin{array}{c}
C_{k}(A)\left(\gamma^{\prime}, \delta\right) \\
C_{k}(A)(\gamma, \delta)
\end{array}\right)=r\left(C_{k}(A)\left(\gamma, \delta^{\prime}\right), C_{k}(A)(\gamma, \delta)\right)=r\left(C_{k}(A) /+(\gamma, \delta)\right)
$$

where $\gamma^{\prime}=\left\{j_{\alpha^{\prime}}^{-\prime}\left|\bar{\alpha}^{\prime} \subset \alpha^{\prime},\right| \bar{\alpha}^{\prime} \mid=k, \overline{\alpha^{\prime}} \in Q_{k, m}\right\}, \delta^{\prime}=\left\{j_{\bar{\beta}^{\prime}}\left|\bar{\beta}^{\prime} \subset \beta^{\prime},\right| \bar{\beta}^{\prime} \mid=k, \bar{\beta}^{\prime} \in\right.$ $\left.Q_{k, n}\right\} ;$ and $\gamma=\left\{1,2, \ldots,\left(\begin{array}{c}m \\ k\end{array}\right)\right\}-\gamma^{\prime}, \delta=\left\{1,2, \ldots,\left(\begin{array}{c}n \\ k\end{array}\right)\right\}-\delta^{\prime}$.

Then

$$
C_{k}[A /+(\alpha, \beta)]=C_{k}(A) /+(\gamma, \delta) .
$$

Proof. For $A \in \mathbb{C}^{m \times n}$, there exist permutation matrices $P \in \mathbb{C}^{m \times m}$ and $Q \in$ $\mathbb{C}^{n \times n}$ such that

$$
P A Q=\left(\begin{array}{cc}
A\left(\alpha^{\prime}, \beta^{\prime}\right) & A\left(\alpha^{\prime}, \beta\right) \\
A\left(\alpha, \beta^{\prime}\right) & A(\alpha, \beta)
\end{array}\right)
$$

Let

$$
\begin{gathered}
\tilde{\alpha^{\prime}}=\left\{1,2, \ldots,\left|\alpha^{\prime}\right|\right\}, \tilde{\beta}^{\prime}=\left\{1,2, \ldots,\left|\beta^{\prime}\right|\right\}, \tilde{\alpha}=M-\tilde{\alpha}^{\prime}, \\
\tilde{\beta}=N-\tilde{\beta^{\prime}}, \tilde{\gamma}^{\prime}=\left\{1,2, \ldots,\left|\gamma^{\prime}\right|\right\}, \tilde{\delta}^{\prime}=\left\{1,2, \ldots,\left|\delta^{\prime}\right|\right\}, \\
\tilde{\gamma}=\left\{1,2, \ldots,\left(\begin{array}{c}
m \\
k
\end{array}\right)\right\}-\tilde{\gamma^{\prime}}, \tilde{\delta}=\left\{1,2, \ldots,\left(\begin{array}{c}
n \\
k
\end{array}\right)\right\}-\tilde{\delta}^{\prime} .
\end{gathered}
$$


Thus, by (2.3), (2.4) and Lemma 2.2, we have

$$
\left[A^{+}\left(\alpha^{\prime}, \beta^{\prime}\right)\right]^{+}=A /+(\alpha, \beta) .
$$

By (2.5), (2.6) and Lemma 2.2, we have

$$
\left\{\left[C_{k}(A)\right]^{+}\left(\gamma^{\prime}, \delta^{\prime}\right)\right\}^{+}=C_{k}(A) /+(\gamma, \delta) .
$$

Therefore, from (1.3), (2.8) and (2.9), it follows that

$$
\begin{aligned}
\left.C_{k}[A]_{+}(\alpha, \beta)\right] & =C_{k}[(P A Q) /+(\tilde{\alpha}, \tilde{\beta})] \\
& =C_{k}\left\{\left[(P A Q)^{+}\left(\tilde{\alpha}, \tilde{\beta}^{\prime}\right)\right]^{+}\right\} \quad(\text { by }(2.8)) \\
& =\left\{C_{k}\left[(P A Q)^{+}\left(\tilde{\alpha}^{\prime}, \tilde{\beta}^{\prime}\right)\right]\right\}^{+} \quad(\text { by }(1.3)) \\
& =\left\{C_{k}\left[(P A Q)^{+}\right]\left(\tilde{\gamma}^{\prime}, \tilde{\delta}^{\prime}\right)\right\}^{+} \\
& =\left\{\left[C_{k}(P A Q)\right]^{+}\left(\tilde{\gamma}^{\prime}, \tilde{\delta}^{\prime}\right)\right\}^{+} \quad(\text { by }(1.3)) \\
& =C_{k}(P A Q) /+(\tilde{\gamma}, \tilde{\delta}) \quad(\text { by }(2.9)) \\
& =C_{k}(A) /+(\gamma, \delta) .
\end{aligned}
$$

Corollary 2.5. Let $A \in \mathbb{C}^{n \times n}, \alpha \subset N$ and $\alpha^{\prime}=N-\alpha$. Set $1 \leq k \leq n-|\alpha|$. If $A, A(\alpha)$, and $A\left(\alpha^{\prime}\right)$ are nonsingular respectively, then

$$
C_{k}(A / \alpha)=C_{k}(A) / \gamma
$$

where $\gamma^{\prime}=\left\{j_{\alpha^{-}}\left|\overline{\alpha^{\prime}} \subset \alpha^{\prime},\right| \overline{\alpha^{\prime}} \mid=k, \bar{\alpha}^{\prime} \in Q_{k, n}\right\}$ and $\gamma=\left\{1,2, \ldots,\left(\begin{array}{c}n \\ k\end{array}\right)\right\}-\gamma^{\prime}$.

In a manner similar to the proof of Theorem 2.4, we obtain the following result by using Lemma 2.3 .

Theorem 2.6. Let $A \in H_{\bar{n}}^{\geq}, \alpha \subset N$ and $\alpha^{\prime}=N-\alpha$. Set $1 \leq k \leq n-|\alpha|$. Suppose that the following conditions are satisfied:

$$
\begin{aligned}
& r(A)=r(A(\alpha))+r\left(A\left(\alpha^{\prime}\right)\right), \\
& r\left[C_{k}(A)\right]=r\left[C_{k}(A)(\gamma)\right]+r\left[C_{k}(A)\left(\gamma^{\prime}\right)\right],
\end{aligned}
$$

where $\gamma^{\prime}=\left\{j_{\alpha^{\prime}}\left|\bar{\alpha}^{\prime} \subset \alpha^{\prime},\right| \overline{\alpha^{\prime}} \mid=k, \overline{\alpha^{\prime}} \in Q_{k, n}\right\}, \gamma=\left\{1,2, \ldots,\left(\begin{array}{c}n \\ k\end{array}\right)\right\}-\gamma^{\prime}$. Then

$$
C_{k}(A /+\alpha)=C_{k}(A) /+\gamma
$$

Corollary 2.7. Let $A \in H_{n}^{>}, \alpha \subset N$ and $\alpha^{\prime}=N-\alpha$. Set $1 \leq k \leq n-|\alpha|$. Then

$$
C_{k}(A / \alpha)=C_{k}(A) / \gamma
$$

where $\gamma^{\prime}=\left\{j_{\alpha^{\prime}}^{-}\left|\overline{\alpha^{\prime}} \subset \alpha^{\prime},\right| \overline{\alpha^{\prime}} \mid=k, \overline{\alpha^{\prime}} \in Q_{k, n}\right\}, \gamma=\left\{1,2, \ldots,\left(\begin{array}{c}n \\ k\end{array}\right)\right\}-\gamma^{\prime}$. 
3. Some Löwner partial orders for compound matrices of sums of matrices. In this section, we obtain some Löwner partial orders for compound matrices of Schur complements of positive semidefinite Hermitian matrices. Further, we obtain some estimates for eigenvalues of Schur complements of sums of positive semidefinite Hermitian matrices.

Lemma 3.1. ([2, p. 184]) Let $A, B \in H_{n}$. Then

$$
\lambda_{t}(A+B) \geq \max _{i+j=n+t}\left\{\lambda_{i}(A)+\lambda_{j}(B)\right\} .
$$

Lemma 3.2. (i) Let $A \in H_{n}^{>}, k \in N$ and $r \in R$. Then

$$
C_{k}\left(A^{r}\right)=\left[C_{k}(A)\right]^{r} .
$$

(ii) Let $A \in H_{\bar{n}}^{\geq}, k \in N$ and $r \in R_{+}$. Then (3.2) holds.

Proof. Since $A \in H_{n}^{>}$, there exists an unitary matrix $U$ such that

$$
A=U \operatorname{diag}\left(\lambda_{1}(A), \ldots, \lambda_{n}(A)\right) U^{*}
$$

where $\lambda_{i}(A)>0(i=1,2, \ldots, n)$. Thus

$$
\begin{aligned}
C_{k}\left(A^{r}\right) & =C_{k}\left[U \operatorname{diag}\left(\lambda_{1}^{r}(A), \ldots, \lambda_{n}^{r}(A)\right) U^{*}\right] \\
& =C_{k}(U) C_{k}\left[\operatorname{diag}\left(\lambda_{1}^{r}(A), \ldots, \lambda_{n}^{r}(A)\right]\left[C_{k}(U)\right]^{*}\right. \\
& =C_{k}(U) \operatorname{diag}\left(\left[\lambda_{1}(A) \ldots \lambda_{k}(A)\right]^{r}, \ldots,\left[\lambda_{n-k+1}(A) \ldots \lambda_{n}(A)\right]^{r}\right)\left[C_{k}(U)\right]^{*} \\
& =\left\{C_{k}(U) \operatorname{diag}\left(\lambda_{1}(A) \ldots \lambda_{k}(A), \ldots, \lambda_{n-k+1}(A) \ldots \lambda_{n}(A)\right)\left[C_{k}(U)\right]^{*}\right\}^{r} \\
& =\left\{C_{k}(U) C_{k}\left[\operatorname{diag}\left(\lambda_{1}(A), \ldots, \lambda_{n}(A)\right)\right]\left[C_{k}(U)\right]^{*}\right\}^{r} \\
& =\left\{C_{k}\left[U \operatorname{diag}\left(\lambda_{1}(A), \ldots, \lambda_{n}(A)\right) U^{*}\right]\right\}^{r} \\
& =\left[C_{k}(A)\right]^{r} .
\end{aligned}
$$

In a manner similar to the proof of (i), we obtain (ii). $\square$

Lemma 3.3. Let $A, B \in H_{\bar{n}}^{\geq}, k \in N$. Then

$$
C_{k}(A+B) \geq C_{k}(A)+C_{k}(B) .
$$

Proof. Since $A, B \in H_{\bar{n}}^{\geq}$, we have

$$
\begin{aligned}
C_{k}(A+B) & =C_{k}\left(A^{\frac{1}{2}} A^{\frac{1}{2}}+B^{\frac{1}{2}} B^{\frac{1}{2}}\right) \\
& =C_{k}\left[\left(A^{\frac{1}{2}}, B^{\frac{1}{2}}\right)\left(A^{\frac{1}{2}}, B^{\frac{1}{2}}\right)^{*}\right] \\
& =C_{k}\left[\left(A^{\frac{1}{2}}, B^{\frac{1}{2}}\right)\right]\left\{C_{k}\left[\left(A^{\frac{1}{2}}, B^{\frac{1}{2}}\right)\right]\right\}^{*} .
\end{aligned}
$$


It is not difficult to show that there exist $X$ and a permutation matrix $U$ such that

$$
C_{k}\left[\left(A^{\frac{1}{2}}, B^{\frac{1}{2}}\right)\right]=\left(C_{k}\left(A^{\frac{1}{2}}\right), C_{k}\left(B^{\frac{1}{2}}\right), X\right) U,
$$

where $X$ is $\left(\begin{array}{c}n \\ k\end{array}\right) \times\left[\left(\begin{array}{c}2 n \\ k\end{array}\right)-2\left(\begin{array}{c}n \\ k\end{array}\right)\right]$ and $U$ is $\left(\begin{array}{c}2 n \\ k\end{array}\right) \times\left(\begin{array}{c}2 n \\ k\end{array}\right)$.

Therefore, by (3.3) and (3.4), we have

$$
\begin{aligned}
C_{k}(A+B) & \left.=\left[\left(C_{k}\left(A^{\frac{1}{2}}\right), C_{k}\left(B^{\frac{1}{2}}\right), X\right) U\right]\left[C_{k}\left(A^{\frac{1}{2}}\right), C_{k}\left(B^{\frac{1}{2}}\right), X\right) U\right]^{*} \\
& =\left(\left[C_{k}(A)\right]^{\frac{1}{2}},\left[C_{k}(B)\right]^{\frac{1}{2}}, X\right)\left(\left[C_{k}(A)\right]^{\frac{1}{2}},\left[C_{k}(B)\right]^{\frac{1}{2}}, X\right)^{*} \\
& =C_{k}(A)+C_{k}(B)+X X^{*} \\
& \geq C_{k}(A)+C_{k}(B) .
\end{aligned}
$$

Lemma 3.4. Let $A, B \in H_{\bar{n}}^{\geq}, A \geq B$ and $k \in N$. Then

$$
C_{k}(A) \geq C_{k}(B) \text {. }
$$

Proof. Lemma 3.3 ensures that

$$
C_{k}(A)=C_{k}[B+(A-B)] \geq C_{k}(B)+C_{k}(A-B) \geq C_{k}(B) .
$$

Theorem 3.5. Let $A, B \in H_{n}^{\geq}, \alpha \subset N$, and $\alpha^{\prime}=N-\alpha$. Set $1 \leq k \leq n-|\alpha|$. Suppose that the following conditions are satisfied:

$$
r\left[C_{k}(A)\right]=r\left[C_{k}(A)(\gamma)\right]+r\left[C_{k}(A)\left(\gamma^{\prime}\right)\right], \quad r\left[C_{k}(B)\right]=r\left[C_{k}(B)(\gamma)\right]+r\left[C_{k}(B)\left(\gamma^{\prime}\right)\right],
$$

where $\gamma^{\prime}=\left\{j_{\bar{\alpha}^{\prime}}\left|\bar{\alpha}^{\prime} \subset \alpha^{\prime},\right| \overline{\alpha^{\prime}} \mid=k, \overline{\alpha^{\prime}} \in Q_{k, n}\right\}$ and $\gamma=\left\{1,2, \ldots,\left(\begin{array}{c}n \\ k\end{array}\right)\right\}-\gamma^{\prime}$. Then

$$
C_{k}[(A+B) /+\alpha] \geq C_{k}(A) /+\gamma+C_{k}(B) /+\gamma .
$$

Proof. By [8, Theorem 3.1], it follows that

$$
(A+B) /+\alpha \geq A /+\alpha+B /+\alpha .
$$

Thus, by (3.5), (3.9), (3.2), (3.6), (3.7) and (2.13), we conclude that

$$
\begin{aligned}
C_{k}[(A+B) /+\alpha] & \geq C_{k}(A /+\alpha+B /+\alpha) \quad(\text { by }(3.5) \text { and }(3.9)) \\
& \geq C_{k}(A /+\alpha)+C_{k}(B /+\alpha) \quad(\text { by }(3.2)) \\
& =C_{k}(A) /+\gamma+C_{k}(B) /+\gamma . \quad(\text { by }(3.6),(3.7) \text { and }(2.13)) .
\end{aligned}
$$


Corollary 3.6. Let all assumptions of Theorem 3.5 be satisfied. If $A-B \in H_{\bar{n}}^{\geq}$, then

$$
C_{k}[(A-B) /+\alpha] \leq C_{k}(A) /+\gamma-C_{k}(B) /+\gamma .
$$

Proof. Since $A-B \in H_{n}^{\geq}$, Theorem 3.5 ensures that

$$
C_{k}(A) /+\gamma=C_{k}(A /+\alpha)=C_{k}[(B+(A-B)) /+\alpha]
$$

is at least

$$
C_{k}(B /+\alpha)+C_{k}[(A-B) /+\alpha]=C_{k}(B) /+\gamma+C_{k}[(A-B) /+\alpha],
$$

which means that (3.11) holds.

TheOREm 3.7. Let all the assumptions of Theorem 3.5 be satisfied. Then

$$
\prod_{t=1}^{k} \lambda_{t}[(A+B) /+\alpha]
$$

is bounded below by the maximum of

$$
\prod_{t=1}^{k} \lambda_{t}\left(A /{ }_{+} \alpha\right)+\prod_{t=1}^{k} \lambda_{n-|\alpha|-t+1}(B /+\alpha)
$$

and

$$
\prod_{t=1}^{k} \lambda_{n-|\alpha|-t+1}(A /+\alpha)+\prod_{t=1}^{k} \lambda_{t}(B /+\alpha)
$$

Proof. Theorem 3.5 and (2.13) imply that

$$
\prod_{t=1}^{k} \lambda_{t}[(A+B) /+\alpha] \lambda_{1}\left\{C_{k}[(A+B) /+\alpha]\right\} \geq \lambda_{1}\left[C_{k}(A /+\alpha)+C_{k}(B /+\alpha),\right.
$$

which is bounded below by the maximum of

$$
\lambda_{1}\left[\left(C_{k}(A /+\alpha)\right]+\lambda(\underset{k}{n-|\alpha|})\left[C_{k}(B /+\alpha)\right]\right.
$$

and

$$
\lambda_{(\underset{k}{n-|\alpha|})}\left[C_{k}(A /+\alpha)+\lambda_{1}\left[\left(C_{k}(B /+\alpha)\right],\right.\right.
$$


and hence by the maximum of

$$
\prod_{t=1}^{k} \lambda_{t}(A /+\alpha)+\prod_{t=1}^{k} \lambda_{n-|\alpha|-t+1}(B /+\alpha)
$$

and

$$
\prod_{t=1}^{k} \lambda_{n-|\alpha|-t+1}(A /+\alpha)+\prod_{t=1}^{k} \lambda_{t}(B /+\alpha)
$$

REMARK 3.8. In a manner similar to the proof of Theorem 3.7, we get the following result

$$
\prod_{t=1}^{k} \lambda_{n-|\alpha|-t+1}[(A+B) /+\alpha] \geq \prod_{t=1}^{k} \lambda_{n-|\alpha|-t+1}(A /+\alpha)+\prod_{t=1}^{k} \lambda_{n-|\alpha|-t+1}(B /+\alpha) .
$$

Theorem 3.9. Let $B \in H_{\bar{n}}^{\geq}, L=\{1,2, \ldots, \min \{m, n\}\}, \alpha \subset L$, and $\alpha^{\prime}=M-\alpha$, $\beta^{\prime}=N-\alpha$. Set $1 \leq k \leq|L-\alpha|$. If $A \in \mathbb{C}^{m \times n}$ satisfies conditions (2.3)-(2.6) and

$$
\Re\left[A\left(\alpha, \alpha^{\prime}\right)\right] \subseteq \Re[A(\alpha)],
$$

then

$$
C_{k}\left[\left(A B A^{*}\right) /+\alpha\right] \leq\left[C_{k}(A) /+\gamma\right] C_{k}(B)\left(\delta^{\prime}\right)\left[C_{k}(A) /+\gamma\right]^{*},
$$

where $\gamma^{\prime}=\left\{j_{\alpha^{-}}\left|\overline{\alpha^{\prime}} \subset \alpha^{\prime},\right| \overline{\alpha^{\prime}} \mid=k, \bar{\alpha}^{\prime} \in Q_{k, m}\right\}, \delta^{\prime}=\left\{j_{\bar{\beta}^{\prime}}\left|\overline{\beta^{\prime}} \subset \beta^{\prime},\right| \overline{\beta^{\prime}} \mid=k, \bar{\beta}^{\prime} \in Q_{k, m}\right\}$, and $\gamma=\left\{1,2, \ldots,\left(\begin{array}{c}m \\ k\end{array}\right)\right\}-\gamma^{\prime}, \delta=\left\{1,2, \ldots,\left(\begin{array}{c}n \\ k\end{array}\right)\right\}-\delta^{\prime}$.

Proof. Using (3.13), in a manner similar to the proof of $[10$, Theorem 3$]$ and $[9$, Theorem 2 ], it follows that

$$
\left(A B A^{*}\right) /{ }_{+} \alpha \leq(A /+\alpha) B\left(\alpha^{\prime}\right)(A /+\alpha)^{*} .
$$

Thus, from (3.15), (3.5) and (2.7), we obtain

$$
\begin{aligned}
C_{k}\left[\left(A B A^{*}\right) /+\alpha\right] & \leq C_{k}\left[(A /+\alpha) B\left(\beta^{\prime}\right)(A /+\alpha)^{*}\right] \quad(\text { by }(3.15) \text { and }(3.5)) \\
& =C_{k}(A /+\alpha) C_{k}\left[B\left(\beta^{\prime}\right)\right] C_{k}\left[(A /+\alpha)^{*}\right] \\
& =\left[C_{k}(A) /+\gamma\right] C_{k}(B)\left(\delta^{\prime}\right)\left[C_{k}(A) /+\gamma\right]^{*} \quad(\text { by }(2.7)) .
\end{aligned}
$$

TheOREM 3.10. Let all assumptions of Corollary 2.7 be satisfied, and $0 \leq l \leq 1$. Then

$$
C_{k}\left(A^{l} / \alpha\right) \geq\left[C_{k}(A) / \gamma\right]^{l}
$$


Proof. By [13], for $1 \leq t \leq+\infty$, we have

$$
A\left(\alpha^{\prime}\right) \leq\left[A^{t}\left(\alpha^{\prime}\right)\right]^{\frac{1}{t}} .
$$

Replace $A$ with $\left(A^{-1}\right)^{\frac{1}{t}}$ in Eqs. (3.17), and let $l=\frac{1}{t}$. Then

$$
\left(A^{l}\right)^{-1}\left(\alpha^{\prime}\right)=\left(A^{-1}\right)^{l}\left(\alpha^{\prime}\right) \leq\left[A^{-1}\left(\alpha^{\prime}\right)\right]^{l} .
$$

It is known by [3, p. 474] that for $B \in H_{n}^{>}$,

$$
B^{-1}\left(\alpha^{\prime}\right)=(B / \alpha)^{-1} \text {. }
$$

Thus, by (3.18) and (3.19), we get

$$
A^{l} / \alpha \geq(A / \alpha)^{l}
$$

Therefore, from (3.20), (3.5), (3.1), and (2.12), we have

$$
C_{k}\left(A^{l} / \alpha\right) \geq C_{k}\left[(A / \alpha)^{l}\right]=\left[C_{k}(A / \alpha)\right]^{l}=\left[C_{k}(A) / \gamma\right]^{l} .
$$

4. Some Löwner partial orders for compound matrices of Schur complements of two types matrices. Let $A \in \mathbb{C}^{n \times n}$. Then

$$
H_{A}=\frac{A+A^{*}}{2}, S_{A}=\frac{A-A^{*}}{2} .
$$

In this section, we study compound matrices of Schur complements of complex square matrices that are either normal or have positive definite Hermitian part.

THEOREM 4.1. Let all assumptions of Corollary 2.5 be satisfied. If $\left(A+A^{*}\right)(\alpha) \in$ $H_{|\alpha|}^{>}$, then

$$
C_{k}\left(A+A^{*}\right) / \gamma \leq C_{k}\left[A / \alpha+(A / \alpha)^{*}\right] \leq\left[\frac{|\operatorname{det} A|}{\operatorname{det} H_{A}}\right]^{2 k} C_{k}\left(A+A^{*}\right) / \gamma .
$$

Proof. By [9, Theorem 7 and Theorem 8], we have

$$
\left[\frac{\operatorname{det} H_{A}}{|\operatorname{det} A|}\right]^{2}\left[A / \alpha+(A / \alpha)^{*}\right] \leq\left(A+A^{*}\right) / \alpha \leq A / \alpha+(A / \alpha)^{*} .
$$

Thus

$$
\left(A+A^{*}\right) / \alpha \leq A / \alpha+(A / \alpha)^{*} \leq\left[\frac{|\operatorname{det} A|}{\operatorname{det} H_{A}}\right]^{2}\left(A+A^{*}\right) / \alpha
$$


By (4.3), (3.5) and (2.10), we get that

$$
\begin{aligned}
C_{k}\left[A / \alpha+(A / \alpha)^{*}\right] & \leq C_{k}\left\{\left[\frac{|\operatorname{det} A|}{\operatorname{det} H_{A}}\right]^{2}\left(A+A^{*}\right) / \alpha\right\} \quad(\operatorname{by}(4.3) \text { and }(3.5)) \\
& =\left[\frac{|\operatorname{det} A|}{\operatorname{det} H_{A}}\right]^{2 k} C_{k}\left[\left(A+A^{*}\right) / \alpha\right] \\
& =\left[\frac{|\operatorname{det} A|}{\operatorname{det} H_{A}}\right]^{2 k} C_{k}\left(A+A^{*}\right) / \gamma \quad(\text { by }(2.10)),
\end{aligned}
$$

and

$$
\begin{aligned}
C_{k}\left[A / \alpha+(A / \alpha)^{*}\right] & \geq C_{k}\left[\left(A+A^{*}\right) / \alpha\right] \\
& =C_{k}\left(A+A^{*}\right) / \gamma .
\end{aligned}
$$

Theorem 4.2. Let $A \in N_{n}, \alpha \subset N$ and $\alpha^{\prime}=N-\alpha$. Set $1 \leq k \leq n-|\alpha|$. Suppose that the following conditions are satisfied:

$$
\begin{aligned}
& r\left(H_{A}^{2}\right)=r\left[H_{A}^{2}(\alpha)\right]+r\left[H_{A}^{2}\left(\alpha^{\prime}\right)\right], \\
& r\left(S_{A}^{2}\right)=r\left[S_{A}^{2}(\alpha)\right]+r\left[S_{A}^{2}\left(\alpha^{\prime}\right)\right], \\
& r\left[C_{k}\left(H_{A}^{2}\right)\right]=r\left[C_{k}\left(H_{A}^{2}\right)(\gamma)\right]+r\left[C_{k}\left(H_{A}^{2}\right)\left(\gamma^{\prime}\right)\right], \\
& r\left[C_{k}\left(S_{A}^{2}\right)\right]=r\left[C_{k}\left(S_{A}^{2}\right)(\gamma)\right]+r\left[C_{k}\left(S_{A}^{2}\right)\left(\gamma^{\prime}\right)\right],
\end{aligned}
$$

where $\gamma^{\prime}=\left\{j_{\alpha^{\prime}}\left|\bar{\alpha}^{\prime} \subset \alpha^{\prime},\right| \bar{\alpha}^{\prime} \mid=k, \bar{\alpha}^{\prime} \in Q_{k, n}\right\}, \gamma=\left\{1,2, \ldots,\left(\begin{array}{c}n \\ k\end{array}\right)\right\}-\gamma^{\prime}$. Then

$$
C_{k}\left[\left(A A^{*}\right) /+\alpha\right] \geq\left[C_{k}\left(H_{A}\right)\right]^{2} /+\gamma+(-1)^{k}\left[C_{k}\left(S_{A}\right)\right]^{2} /+\gamma .
$$

Proof. Since $A \in N_{n}$, we have

$$
H_{A} S_{A}=S_{A} H_{A} .
$$

Noting that $S_{A}^{*}=-S_{A}$, by (4.6), we obtain

$$
\begin{gathered}
A A^{*}=\left(H_{A}+S_{A}\right)\left(H_{A}+S_{A}\right)^{*}=\left(H_{A}+S_{A}\right)\left(H_{A}+S_{A}^{*}\right) \\
=H_{A}^{2}+H_{A} S_{A}^{*}+S_{A} H_{A}+S_{A} S_{A}^{*}=H_{A}^{2}+S_{A} S_{A}^{*} .
\end{gathered}
$$

By (4.6), (3.9), (3.5), (3.2) and (2.11), we have

$$
C_{k}\left[\left(A A^{*}\right) /{ }_{+} \alpha\right]=C_{k}\left[\left(H_{A}^{2}+S_{A} S_{A}^{*}\right) /+\alpha\right] \quad(\text { by }(4.6))
$$




$$
\begin{aligned}
& \geq C_{k}\left[H_{A}^{2} /+\alpha+\left(S_{A} S_{A}^{*}\right) /+\alpha\right] \quad(\text { by }(3.9) \text { and }(3.5)) \\
& \geq C_{k}\left(H_{A}^{2} /+\alpha\right)+C_{k}\left[\left(S_{A} S_{A}^{*}\right) /+\alpha\right] \quad(\text { by }(3.2)) \\
& =C_{k}\left(H_{A}^{2}\right) /+\gamma+C_{k}\left(S_{A} S_{A}^{*}\right) /+\gamma \quad(\text { by }(2.11)) \\
& =\left[C_{k}\left(H_{A}\right)\right]^{2} /+\gamma+C_{k}\left(-S_{A}^{2}\right) /+\gamma \\
& =\left[C_{k}\left(H_{A}\right)\right]^{2} /+\gamma+(-1)^{k}\left[C_{k}\left(S_{A}\right)\right]^{2} /+\gamma
\end{aligned}
$$

ThEOREM 4.3. Let all the assumptions of Corollary 2.5 be satisfied. Suppose $A \in N_{n}$ is nonsingular and each of $H_{A}$ and $S_{A}$ is nonsingular. Then

$$
\begin{aligned}
C_{k}\left[\left(A A^{*}\right)^{-1} / \alpha\right] \geq & {\left[C_{k}\left(H_{A}-S_{A} H_{A}^{-1} S_{A}\right)\right]^{-2} / \gamma } \\
& +(-1)^{k}\left[C_{k}\left(S_{A}-H_{A} S_{A}^{-1} H_{A}\right)\right]^{-2} / \gamma .
\end{aligned}
$$

Proof. Since $A \in N_{n}$, we have $A^{-1} \in N_{n}$. Further

$$
\begin{aligned}
H_{A^{-1}} & =\frac{1}{2}\left(A^{-1}+\left(A^{-1}\right)^{*}\right) \\
& \left.=\frac{1}{2} A^{-1}\left(A+A^{*}\right)\left(A^{-1}\right)^{*}\right) \\
& =\left(A^{*} H_{A}^{-1} A\right)^{-1} \\
& =\left[\left(H_{A}+S_{A}\right)^{*} H_{A}^{-1}\left(H_{A}+S_{A}\right)\right]^{-1} \\
& =\left(H_{A}-S_{A} H_{A}^{-1} S_{A}\right)^{-1} .
\end{aligned}
$$

Similarly, we have

$$
S_{A^{-1}}=\frac{1}{2}\left(A^{-1}-A^{-1 *}\right)=\left(S_{A}-H_{A} S_{A}^{-1} H_{A}\right)^{-1} .
$$

Thus, in a manner similar to the proof of Theorem 4.2, we obtain (4.8).

5. Conclusions. We have obtained some formulae for compound matrices of generalized Schur complements of matrices. Using these results, we studied some Löwner partial orders for compound matrices of Schur complements of positive semidefinite Hermitian matrices. If $A, B \in H_{\bar{n}}^{\geq}$, we extend some results in [1] and show that

$$
C_{k}[(A+B) /+\alpha] \geq C_{k}(A) /+\gamma+C_{k}(B) /+
$$

under some restrictive conditions, as shown in Theorem 3.5, as well as

$$
C_{k}\left(A^{l} / \alpha\right) \geq\left[C_{k}(A) / \gamma\right]^{l}
$$

if $A \in H_{n}^{>}$, as shown in Theorem 3.10. In addition, we provide some results for compound matrices of Schur complements of complex square matrices that are either 
normal or have positive definite Hermitian part. We obtained some estimates for eigenvalues.

Acknowledgment. The authors thank the referee for many valuable and detailed suggestions, which have led to an improvement in the presentation of this paper.

\section{REFERENCES}

[1] T. Ando. Concavity of certain maps on positive definite matrices and applications to Hadamard Products. Linear Algebra Appl., 26:203-241, 1979.

[2] F. Burns, D. Carlson, E. Haynsworth, T. Markham. Generalized inverse formulas using the Schur complement. SIAM J. Appl. Math., 26:254-257, 1974.

[3] R.A. Horn, C.R. Johnson. Matrix Analysis. Cambridge University Press, New York, 1985.

[4] C.K. Li, R. Mathias. Extremal characterizations of the Schur complement and resulting inequalities. SIAM Review, 42(2):233-246, 2000.

[5] J.Z. Liu. Some inequalities for singular values and eigenvalues of generalized Schur complements of products of matrices. Linear Algebra Appl., 286:209-221, 1999.

[6] J.Z. Liu, L. Zhu. A minimum principle and estimates of the eigenvalues for Schur complements of positive semidefinite Hermitian matrices. Linear Algebra Appl., 265:123-145, 1997.

[7] J.Z. Liu, J. Wang. Some inequalities for Schur complements. Linear Algebra Appl., 293:233-241, 1998.

[8] J.Z. Liu. Some Löwner partial orders of Schur complements and Kronecker products of matrices. Linear Algebra Appl., 291:143-149, 1999.

[9] A.W. Marshall, I. Olkin. Inequalities: Theory of majorization and its Applications. Academic Press, New York, 1979.

[10] D.V. Ouellette. Schur complements and statistics. Linear Algebra Appl., 36:254-257, 1981.

[11] R.L. Smith. Some interlacing properties of Schur complements of a Hermitian matrix. Linear Algebra Appl., 177:137-144, 1992.

[12] B.Y. Wang, X. Zhang, F. Zhang. Some inequalities on generalized Schur complements. Linear Algebra Appl., 302-303:163-172, 1999.

[13] B.Y. Wang, F. Zhang. Schur complements and matrix inequalities of Hadamard products. Linear and Multilinear Algebra, 43:315-149, 1997. 